\title{
Un esempio di introduzione del paradigma relazionale nella scuola media
}

\section{An example of introduction of the relational paradigm in lower secondary school}

\author{
Alice Lemmo ${ }^{\circ}$ e Andrea Maffia ${ }^{\circ}$ \\ - Università dell’Aquila - Italia \\ - Università di Pavia - Italia \\ 凶 alice.lemmo@univaq.it, andrea.maffia@unipv.it
}

Sunto / La rappresentazione simbolica di relazioni tra numeri viene spesso introdotta nella scuola media. Vari autori sostengono che l'adozione di un paradigma relazionale e l'uso di rappresentazioni grafiche possono supportare gli studenti nel primo approccio all'algebra, tuttavia esempi di sequenze didattiche del genere non sono ancora largamente diffusi. Si propone un esempio ispirato al lavoro di Castellini (2016) e, all'interno di un design quasi-sperimentale, se ne valuta l'efficacia usando uno strumento di valutazione appositamente sviluppato che comprende tutte e cinque le componenti dell'apprendimento della matematica di Fandiño Pinilla. Si nota una particolare efficacia in termini di apprendimento strategico e significativi progressi in termini comunicativi.

Parole chiave: relazioni; scuola media; algebra; valutazione.

\begin{abstract}
The symbolic representation of relations between numbers is often introduced in lower secondary school. Many authors claim that a relational paradigm and the use of graphical representations may support students in approaching algebra. However, examples of such teaching sequences are still not widespread in schools. We propose an example inspired by Castellini's work (Castellini, 2016) and adopting a quasi-experimental design we evaluate its effectiveness. An ad hoc instrument for assessment has been develop considering the five components of mathematical learning by Fandiño Pinilla. Data show a specific effectiveness in relation to problem solving, and significant progress in communicational learning.
\end{abstract}

Keywords: relations; lower secondary school; algebra; assessment.

La scuola media è sicuramente il livello scolastico in cui lo studente fa il suo primo incontro formale con il linguaggio dell'algebra come strumento di rappresentazione di relazioni che intercorrono tra quantità incognite e/o variabili. Come si legge nel Piano di studio della scuola dell'obbligo ticinese

(C) 2021 Lemmo Alice e Maffia Andrea. Questo è un articolo Open Access, sottoposto a un processo di revisione tra pari a doppio cieco, pubblicato dal Centro competenze didattica della matematica e dal Servizio risorse didattiche e scientifiche, eventi e comunicazione del DFA-SUPSI in collaborazione con il DECS. L'articolo è distribuito sotto i termini della Licenza Creative Commons Attribuzione 4.0 Internazionale (CC BY 4.0) che permette di usare, condividere e modificare l'articolo su qualsiasi mezzo a patto che I'autore e la fonte originale siano citati. 
«ll calcolo algebrico viene proposto come generalizzazione del calcolo aritmetico. Grazie ad esso si intendono sviluppare negli allievi le competenze necessarie per gestire situazioni problema risolvibili tramite espressioni aritmetiche, equazioni, [...] secondo varie modalità e sfruttando le proprietà del calcolo, riuscendo così a matematizzare e modellizzare la realtà».

(Dipartimento dell'educazione, della cultura e dello sport [DECS], 2015, p. 153)

Riferimenti analoghi si possono rintracciare nelle Indicazioni Nazionali del primo ciclo italiane; tra gli obiettivi alla conclusione del primo ciclo, si legge: «Interpretare, costruire e trasformare formule che contengono lettere per esprimere in forma generale relazioni e proprietà» (Ministero dell'Istruzione, dell'Università e della Ricerca [MIUR], 2012, p. 54).

La letteratura in didattica della matematica ha ampiamente mostrato come un approccio prettamente sintattico all'introduzione della lettera possa risultare infruttuoso (Siety, 2001/2003). In alternativa, viene proposto un graduale avvicinamento all'algebra attraverso attività volte alla generalizzazione di proprietà osservate in ambito aritmetico (Radford, 2010). L'insieme di questi approcci prende il nome di early algebra, ne è un esempio a livello italiano il progetto ArAl (Navarra, 2019). Nonostante le evidenze scientifiche circa l'efficacia di queste prospettive, la loro diffusione all'interno delle pratiche scolastiche sembra ancora non essere così vasta, forse anche per l'altrettanto scarsa diffusione di esempi di sequenze didattiche e per la mancanza di prove di valutazione coerenti.

Un esempio di approccio all'uso della lettera per rappresentare relazioni tra numeri (naturali) e operazioni è fornito da Castellini (2016). Si tratta di esempi di attività svolte in classe da un'insegnante di scuola media (l'autrice) a partire dalla rielaborazione personale della letteratura di ricerca, corredati dalla riflessione della stessa docente sull'efficacia didattica dell'approccio.

I problemi verbali che esprimono relazioni tra quantità vengono generalmente proposti agli studenti con tecniche di risoluzione attraverso un metodo grafico; per questo motivo vengono spesso nominati "problemi con i segmenti". Secondo l'autrice, questo genere di approccio pone «l'accento sulla rappresentazione grafica mentre la difficoltà [...] è a monte ovvero nella identificazione, comprensione, appropriazione delle informazioni di tipo relazionale» (Castellini, 2016, p. 289). Castellini sottolinea che, in una situazione problematica, un dato assoluto è generalmente ben identificato dagli studenti (Pierino possiede tot mele; il perimetro del triangolo è pari a...; ...) mentre invece il dato relazionale (il doppio; la metà; il successivo; ...) sfugge spesso alla loro attenzione. Nel suo lavoro, Castellini introduce una rappresentazione grafica intermedia (nel senso di Davydov, 1982) tra la situazione problematica affrontata e il simbolismo algebrico. In questa rappresentazione, le quantità note sono rappresentate mediante palline (presentate come oggetti concreti o disegnate come cerchietti), ciascuna delle quali ha valore 1. Le quantità incognite sono invece rappresentate mediante barattoli (rappresentati da contenitori concreti o disegnati a forma di cilindri) che, convenzionalmente, contengono tutti la stessa quantità (non nota) di palline. Per esempio, in Figura 1 è rappresentata l'espressione $2 x+3$.

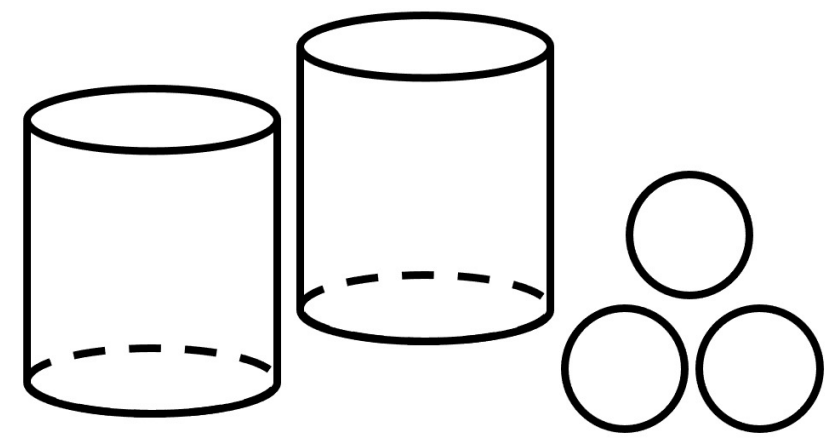

Figura 1. Esempio di rappresentazione di $2 x+3$ secondo la proposta di Castellini (2016). 
Questo approccio nasce dall'attenzione verso gli sviluppi storico-epistemologici che hanno portato alla oggettivazione del linguaggio algebrico come oggetto matematico (Radford, 2008), riprendendo I'idea di "mucchio" proposta dagli antichi egizi (si veda il par. 2). Nel prossimo paragrafo di questo contributo presentiamo un'analisi a priori dell'approccio proposto da Castellini (2016) in cui evidenziamo le sue potenzialità didattiche facendo riferimento alla letteratura in didattica della matematica. Seguirà poi una valutazione a posteriori della possibile efficacia di un simile approccio all'introduzione dell'uso della lettera nella scuola media. Si tratta di uno studio con un design quasi-sperimentale condotto in classe prima e per il quale è stata appositamente ideata una prova di valutazione che sarà presentata di seguito.

\section{Potenzialità didattiche dell'approccio}

Il Piano di studio della scuola dell'obbligo ticinese si sviluppa in tre cicli che vanno dal primo all'undicesimo anno di scolarità. Per quanto riguarda la matematica, essi si articolano in cinque ambiti di competenza: "Numeri e calcolo", "Geometria", "Grandezze e misure" (dal primo ciclo), "Funzioni" e "Probabilità e statistica" (dal terzo ciclo). L'aritmetica viene quindi esplorata sin dai primi anni di scolarità, ma l'algebra come strumento per generalizzare fatti aritmetici e modellizzare la realtà viene introdotta esplicitamente solo nella scuola media. Questo, però, non significa che l'insegnamento dell'aritmetica nei primi cicli non possa dare spazio ad attività in ambito numerico, di tipo rappresentativo o relazionale, che guidino gli allievi a riconoscere analogie fra strutture aritmetiche, a vedere il generale nel particolare.

Nei traguardi di competenza del piano di studio sono presentati gli obiettivi irrinunciabili che gli studenti dovrebbero raggiungere alla conclusione di ciascun ciclo di istruzione; lo studio dell'aritmetica comincia dai numeri naturali nel primo ciclo e si conclude con i numeri reali nel terzo ciclo. Questa gradualità mette in risalto una scelta esplicita di passaggio dal discreto al continuo; esso si riscontra in parallelo anche nel processo di misura che passa da un confronto tra una grandezza e un'unità scelta, all'utilizzo di strumenti che prevedano I'uso di scale graduate (un discorso analogo può essere fatto nel caso italiano). Gli studi di Fischbein (1987) mostrano che i primi modelli didattici che vengono presentati agli allievi sembrano diventare profondamente radicati e continuano a esercitare un controllo inconscio sul comportamento degli allievi anche da adulti; per esempio, l'introduzione dell'addizione ripetuta come modello della moltiplicazione porta spesso a pensare che il prodotto debba essere sempre maggiore del moltiplicando (Fischbein et al., 1985). L'autore chiama questi modelli primitivi poiché si costruiscono nei primi anni di scolarità e continuano a permanere anche dopo che l'allievo ha acquisito nozioni matematiche formali, solide e corrette. Conoscere i modelli primitivi può essere importante per l'interpretazione e l'intervento su possibili misconcezioni o difficoltà che possono incontrare gli alunni (Sbaragli, 2005). In base alle indicazioni dei piani di studio, infatti, è possibile che gli studenti costruiscano i primi modelli di numero nel discreto, a partire cioè dai numeri naturali, per poi passare al continuo, ovvero ai numeri reali. Questa osservazione potrebbe indicare che, anche nel costruire relazioni tra numeri, i modelli a cui gli alunni fanno riferimento potrebbero inizialmente essere nell'ambito dei numeri naturali per poi essere ampliati agli altri insiemi numerici.

L'approccio iniziale al discreto piuttosto che al continuo è oggetto di discussione nella letteratura in didattica della matematica (si veda per esempio Mellone et al., 2020). In particolare, la precoce introduzione al continuo è sostenuta dai ricercatori che fanno riferimento al lavoro seminale dal matematico russo Davydov (1982), il quale vede nelle relazioni il concetto matematico fondamentale, che dovrebbe essere insegnato addirittura prima delle operazioni (Polotskaia \& Savard, 2021). All'interno del paradigma relazionale «il concetto di numero emerge dal confronto moltiplicativo di due gran- 
dezze (o quantità incognite), una che gioca il ruolo di unità di misura mentre l'altra viene misurata» (Polotskaia \& Savard, 2021, p. 449, traduzione degli autori).

Possiamo osservare che nell'esempio riportato in Figura 1, il ruolo assunto dai barattoli (cilindretti) nella rappresentazione è proprio quello di unità di misura ${ }^{1}$ che permette il confronto moltiplicativo tra quantità indefinite. In altre parole, il bicchiere, che rappresenta l'incognita, può essere percepito dell'allievo come un contenitore di numero incognito di elementi discreti; al contrario il segmento potrebbe essere inteso come «un pezzo di retta che contiene centimetri» (Castellini, 2016, p. 292) e quindi una rappresentazione continua di quantità. Questo risulta ancora più chiaro in altri esempi proposti da Castellini (2016) come quello mostrato in Figura 2, in cui viene chiesto agli studenti di descrivere la relazione tra i bicchieri di Anna e quelli di Bea in termini di doppio o metà.

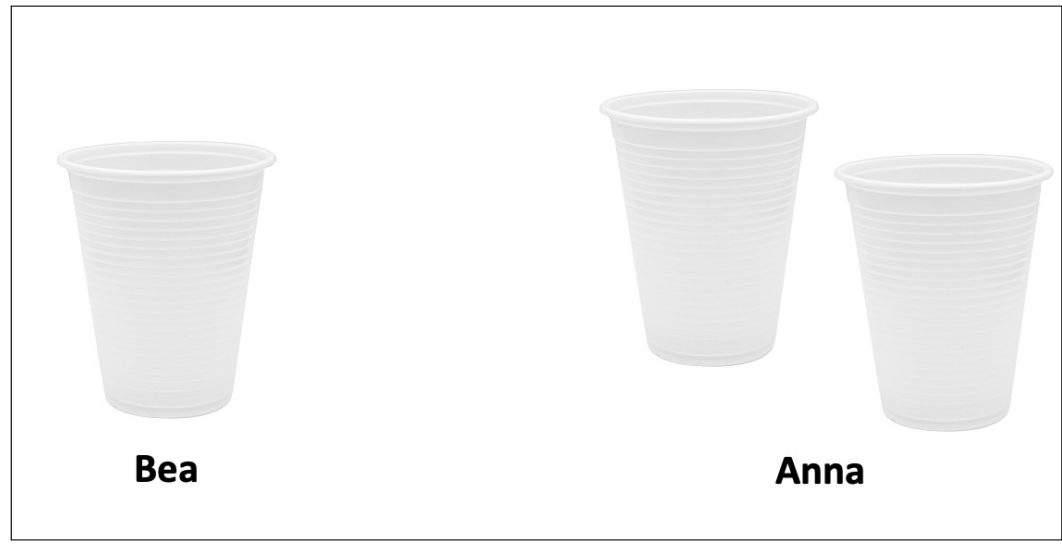

Figura 2. Esempio di confronto moltiplicativo tra quantità incognite. Immagine tratta da Castellini (2016, p. 292).

L'uso di contenitori opachi è voluto allo scopo di evitare che le quantità utilizzate nella rappresentazione non siano individuabili ma restino non conosciute. Questa scelta permette di rappresentare la quantità incognita e confrontarle in situazioni che altrimenti potrebbero essere difficilmente gestibili. L'obiettivo è evitare che gli studenti operino con quantità determinate ma trattino il contenuto del bicchiere come una variabile. In letteratura, si parla di lack of closure (mancanza di chiusura) definito da Collis (1974, citato in Kieran, 1981) per riferirsi alla necessità di sostituire sempre a un'operazione il suo risultato, che comporta l'impossibilità di gestire un calcolo che non può effettivamente essere svolto. Probabilmente non è un caso che la stessa Castellini (2016) riconosca nell'origine dell'idea di usare dei contenitori l'analogo espediente utilizzato nel papiro di Ahmes (risalente al 1550 a.C.) per gestire le quantità incognite. Nel papiro, le quantità incognite sono designate dalla parola "aha", traducibile come "mucchio" (Boyer, 1968/2017; D'Amore \& Sbaragli, 2017). Viene per esempio utilizzato per enunciare situazioni problematiche del tipo "[qual è] il valore del mucchio se il mucchio e un settimo del mucchio sono uguali a 19» (Boyer, 1968/2017, p. 23), in cui svolge il ruolo della quantità incognita. II termine mucchio (in modo del tutto analogo ai contenitori impiegati da Castellini) permette contemporaneamente di designare una moltitudine e un singolo oggetto (tant'è che la parola è usata al singolare) permettendo quindi di "misurare" una quantità totale in termini di un'altra quantità incognita, così come auspicato da Davydov (1982). Un ulteriore vantaggio è rappresentato dalla possibilità di inserire effettivamente delle palline (o dei ceci, come suggerisce Castellini, 2016) all'interno di contenitori opachi e tappati in modo che lo studente possa percepire la presenza

1. Qui il termine "unità di misura" deve intendersi nel senso di "composite unit" così come definito da Steffe (1994, p. 15): «a unit that itself is composed of units». L'autore sostiene che la differenza tra il pensiero additivo e quello moltiplicativo sta proprio nel passare da unità a unità composite, pensare al moltiplicando come un'unità (composita) che si ripete. Per esempio, in $7 \times 3$ il 7 può essere visto come l'unità composita (composta di 7 unità) che viene ripetuta 3 volte. 
di una quantità di oggetti in questo "mucchio". Dobbiamo notare che in questo modo si restringe la possibilità d'uso di questa rappresentazione a sole relazioni tra numeri naturali. L'uso di liquidi o della sabbia, al posto delle palline, potrebbe permettere un passaggio al continuo (Davydov, 1982). Ancora, alte numerosità di piccoli oggetti discreti potrebbero spingere a trattare le quantità discrete come se fossero continue (Mellone et al., 2020).

L'approccio proposto da Castellini (2016) non è interessante solo per la rappresentazione mediante palline e barattoli, ma anche per via delle tipologie di consegne proposte agli studenti. Nel suo articolo si fa riferimento a consegne del tipo «descrivi a parole la situazione che vedi» (Castellini, 2016, p. 292) in cui si invita esplicitamente lo studente alla conversione in linguaggio quotidiano della relazione rappresentata con barattoli e palline. Questo appare di rilevante importanza sia per acquisire I'abitudine di utilizzare certe rappresentazioni come possibile traduzione di situazioni espresse verbalmente (ovvero nel caso dei problemi espressi a parole), sia per l'obiettivo più generale di distinguere l'oggetto matematico "relazione" dalla particolare rappresentazione. Secondo Duval:

«[...] la matematica, sia il suo insegnamento che le pratiche più avanzate, è il campo in cui l'uso dei segni è il più complesso e la gamma eterogenea di segni usati è la più estesa. Questo è dimostrato essere così intrinseco dell'attività matematica che possiamo permetterci di affermare che non c'è pensiero matematico senza usare rappresentazioni semiotiche per trasformarle in altre rappresentazioni semiotiche».

(Duval, 2008, p. 39, traduzione degli autori)

Ci si potrebbe tuttavia chiedere se la sola conversione dal linguaggio quotidiano spontaneo a quello simbolico-algebrico (e viceversa) non possa essere sufficiente. Come sostengono Bazzini e laderosa (2000), il linguaggio algebrico, se da una parte eclissa la semantica espressa dal linguaggio verbale, dall'altra agevola ragionamenti di tipo più avanzato. L'evoluzione storica della disciplina testimonia le difficoltà incontrate nel passaggio dalla risoluzione di problemi con procedimenti espressi a parole (algebra retorica) all'introduzione e all'uso consapevole di simboli (algebra sincopata, mista di simboli e parole, e poi algebra simbolica).

Secondo vari autori, gli studenti incontrano spesso difficoltà nel modellizzare situazioni problematiche (descritte a parole) direttamente in forma di equazione; un modello grafico di tipo analogico può svolgere un importante ruolo fornendo una forma intermedia di modellizzazione (Cai et al., 2005; Polotskaia \& Savard, 2021; Xin et al., 2011). Polotskaia e Savard (2021) analizzano una rappresentazione proposta da Van de Walle e Lovin (2008, citati in Polotskaia \& Savard, 2021) del tutto analoga a quella proposta da Castellini (2016). In riferimento alla rappresentazione della situazione problematica (Figura 3) «Roberto e Anna hanno 15 libri; Roberto ha il doppio dei libri di Anna. Quanti libri ha ciascuno di loro?» ne descrivono così le potenzialità:

«Essa mostra ripetizioni di insiemi uguali all'insieme di riferimento e usa i numeri e la notazione analogico-visuale per indicare la relazione di comparazione. Essa non mostra elementi dei singoli insiemi (nessun libro è rappresentato) e quindi può rappresentare quantità incognite. [...] Da questa combinazione di rappresentazioni, il solutore del problema verbale può scoprire visivamente $^{2}$ che il totale dei libri è composto da 3 parti uguali e quindi risolvere il problema».

(Polotskaia \& Savard, 2021, p. 456, traduzione degli autori)

2. Il grassetto è stato mantenuto come da originale. 


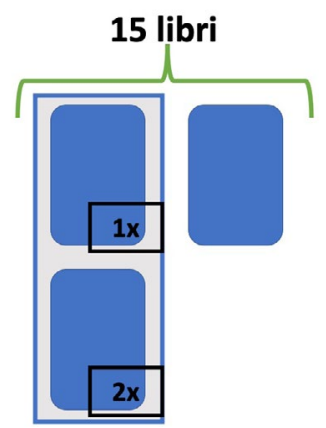

Figura 3. Esempio di rappresentazione proposta da Van de Walle e Lovin (2008, citati in Polotskaia \& Savard, 2021, p. 456).

Più in generale, le stesse autrici (Polotskaia \& Savard, 2021) suggeriscono che una rappresentazione che vuole supportare lo sviluppo del pensiero relazionale attraverso la soluzione di problemi debba soddisfare tre requisiti:

- la rappresentazione può presentare sia le quantità note sia quelle incognite;

- la rappresentazione mostra il ruolo di ciascun elemento all'interno della relazione;

- la rappresentazione supporta l'analisi visiva e la scoperta di nuove informazioni.

Abbiamo notato nella sezione precedente che la prima condizione è soddisfatta dalla rappresentazione con barattoli e palline. L'analisi di Polotskaia e Savard citata mostra come anche gli altri due requisiti siano soddisfatti.

\title{
3 Quadro teorico
}

L'obiettivo di questo lavoro è quello di studiare l'efficacia di un approccio didattico per l'insegnamento/apprendimento di un particolare contenuto matematico; in questo caso la gestione delle relazioni aritmetiche tra numeri naturali. Per fare questo è necessario chiarire cosa si intende per insegnamento/apprendimento della matematica.

Per descrivere i diversi fenomeni tipici dell'insegnamento/apprendimento della matematica, Fandiño Pinilla sostiene che:

\begin{abstract}
«L'apprendimento della matematica si presenta come un fattore multiplo, ricco di mille aspetti: è sotto gli occhi di tutti gli insegnanti il fatto che un apprendimento riuscito in matematica è da considerarsi un'ottimale combinazione di apprendimenti specifici e distinti. In matematica, infatti, non basta aver costruito un concetto, ma occorre saperlo usare per effettuare calcoli o dare risposta a esercizi, combinarlo con altri e con strategie opportune per risolvere problemi, occorre saper spiegare a sé stessi e agli altri il concetto costruito e la strategia seguita, occorre saper far uso sapiente delle trasformazioni semiotiche che permettono di passare da una rappresentazione ad un'altra».
\end{abstract}

(Fandiño Pinilla, 2015, p. 10)

Secondo l'autrice, appoggiare su queste categorie l'azione di ricerca didattica può fornire all'insegnante un riferimento utile a organizzare meglio il proprio lavoro, coordinando l'insegnamento con gli obiettivi di apprendimento. In questa prospettiva Fandiño Pinilla (2008) propone una descrizione dell'apprendimento della matematica multidimensionale, composto da almeno cinque tipologie di apprendimenti distinti che fanno capo a diversi ambiti cognitivi: 
- apprendimento concettuale (noetica);

- apprendimento algoritmico (calcolare, operare ecc.);

- apprendimento strategico (risolvere, congetturare ecc.);

- apprendimento comunicativo (dire, argomentare, validare, dimostrare ecc.);

- apprendimento e gestione delle trasformazioni semiotiche (di trattamento e di conversione).

Prima di descrivere nel dettaglio ciascuna componente, l'autrice sottolinea più volte che queste componenti dell'apprendimento non sono indipendenti, separabili, ma interagiscono tra loro attraverso il meccanismo dell'intersezione, come se si permeassero vicendevolmente.

L'apprendimento concettuale non si riferisce ad un apprendimento ancorato alla conoscenza delle definizioni e proprietà degli oggetti matematici, ma un apprendimento relativo ad una riflessione critica sul perché, sul motivo di determinati procedimenti, definizioni, formalismi ecc. Esso, dunque, riguarda i concetti, visti come la conoscenza e la padronanza di determinate nozioni.

L'apprendimento algoritmico riguarda invece le procedure che vengono messe in campo nel affrontare situazioni matematiche. Secondo l'autrice i passaggi da effettuare nel percorso algoritmico sono meccanici, ma essi vengono effettuati dall'individuo se e solo se egli sa che cosa sta facendo e perché; ogni passo "meccanico" ha una sua funzione ed una sua giustificazione logica e concettuale.

In questa prospettiva, è bene dare importanza a situazioni più problematiche nelle quali la gestione degli algoritmi non sia vista come meramente esecutiva.

L'apprendimento strategico si riferisce ai processi legati al porsi, affrontare e risolvere problemi. Tali problemi possono essere presentati in contesto matematico o reale. L'autrice descrive l'apprendimento strategico attraverso ulteriori sotto-processi: comprensione del testo, trasformazione o traduzione dell'enunciato in una forma aritmetica, scelta e uso delle strategie e, infine, validazione della risposta. In riferimento al processo di traduzione e trasformazione, possiamo riprendere la definizione di matematizzazione (che trae origine dalla teoria della Realistic Mathematics Education introdotta da Freudenthal): Treffers (1987) distingue la matematizzazione orizzontale come il passaggio e la comunicazione tra due mondi (reale e matematico) e la matematizzazione verticale, invece, come l'elaborazione di strategie e procedure all'interno di uno stesso mondo.

L'apprendimento comunicativo si riferisce alla capacità di esprimere idee matematiche, giustificando, argomentando, dimostrando e facendo uso di figure, disegni o schemi per comunicare. Spiegazione e giustificazione sono i due processi principalmente coinvolti nella scuola media; Brunero e Panero (2019) propongono una distinzione tra spiegazione e giustificazione (a partire da precedenti studi come quello di Yackel, 2001) a seconda delle funzioni che esse svolgono. Si parla di spiegazione nel momento in cui l'alunno è chiamato a

«[...] chiarire aspetti del proprio ragionamento matematico che potrebbero non risultare evidenti agli altri. In casi specifici, tale spiegazione svolge la funzione di giustificazione se è fornita per reagire alle contestazioni di apparenti violazioni dell'attività normativa matematica. Una giustificazione matematica è quindi un caso particolare di spiegazione matematica che fa riferimento più direttamente a proprietà matematiche o criteri condivisi».

(Brunero \& Panero, 2019, p. 84)

Infine l'apprendimento legato alle trasformazioni semiotiche, riguarda la gestione di tutte le diverse trasformazioni che si possono utilizzare di uno stesso oggetto matematico attraverso uno o più sistemi di segni (Duval, 2008). La ricerca in didattica della matematica pone l'accento sul non dare per scontate le trasformazioni semiotiche, bisogna infatti abituare gli studenti al fatto che le diverse rappresentazioni di uno stesso oggetto siano equivalenti ed univoche. 


\section{Metodologia della ricerca}

Lo studio dell'efficacia dell'intervento in classe è stato realizzato adottando un approccio quasi-sperimentale classico. Si adotta un disegno quasi-sperimentale perché non è possibile controllare tutti i fattori per cui differiscono le classi oltre all'intervento sperimentale (insegnanti differenti, numero di alunni per classe, composizione delle classi ecc.). Si sono quindi individuati un gruppo sperimentale e un gruppo di controllo e raccolti dati sia prima che dopo l'intervento sperimentale. I dati di pre- e post-test dei due gruppi (sperimentale e controllo) vengono quindi messi a confronto.

\subsection{Contesto di ricerca}

Tutte e cinque le classi prime di una scuola secondaria di primo grado ${ }^{3}$ di Ravenna sono state coinvolte nel progetto, per un totale di 102 studenti. Tre delle classi (64 studenti) hanno svolto il percorso sperimentale proposto (par. 4.2); altre due classi (38 studenti) sono servite da gruppo di controllo. Tutte le classi hanno svolto la stessa prova (par. 4.3) come pre-test e post-test. La sperimentazione è stata condotta nell'anno scolastico 2020-21 e ha avuto una durata di cinque settimane; il campione scelto è un campione di convenienza. Ciascuna delle classi ha una diversa insegnante di matematica, ma in tutte le classi è in uso lo stesso libro di testo; mentre il gruppo sperimentale ha seguito l'intervento descritto nella prossima sezione, gli studenti del gruppo di controllo hanno seguito la proposta presente su tale libro di testo in cui viene introdotto l'uso di segmenti per rappresentare quantità incognite nella soluzione di problemi. Un esempio riportato nel libro di testo è mostrato in Figura 4. Gli studenti del gruppo di controllo hanno svolto anche a casa esercizi tratti dal loro libro di testo.

\section{Esempi॰}

Al campo estivo ci sono 32 ragazzi. I maschi sono 6 in più rispetto alle femmine.

a) Quante sono le femmine?

b) E i maschi?

Si conosce il numero totale di ragazzi, ma non si conosce né il numero di maschi né il numero di femmine. Può essere d'aiuto disegnare uno schema del problema utilizzando i segmenti.

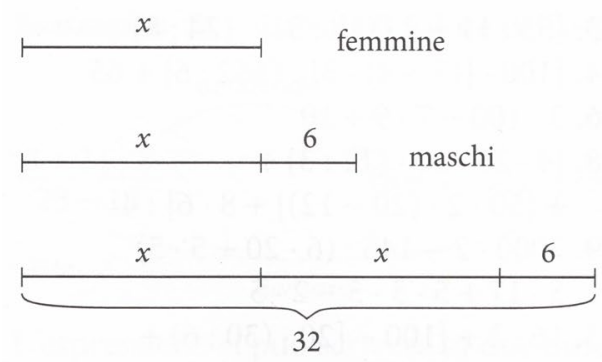

a) Modo 1

Si può impostare un'espressione

$$
\begin{aligned}
& \frac{(32-6)}{2}= \\
& =\frac{26}{2}=13
\end{aligned}
$$

$$
\begin{aligned}
& \text { Modo } 2 \\
& \text { Si può anche risolvere per fasi } \\
& 32-6=26 \\
& 26: 2=13
\end{aligned}
$$

b) Il numero di maschi si trova con l'addizione $13+6=19$ oppure con la sottrazione $32-13=19$.

Risposta:
a) 13 femmine
b) 19 maschi. 
Le insegnanti del gruppo sperimentale hanno ricevuto i materiali presentati di seguito ed è stato proposto loro di leggerli autonomamente. Successivamente, un incontro tra gli autori e le insegnanti è servito a chiarire eventuali dubbi sull'implementazione. Le insegnanti non hanno ricevuto ulteriore formazione sul paradigma relazionale, né è stato presentato loro il lavoro di Castellini (2016).

\subsection{L'intervento sperimentale}

L'intervento sperimentale è stato presentato alle insegnanti coinvolte nello studio attraverso sei schede di lavoro per lo studente. Ciascuna scheda è stata accompagnata dalla relativa guida per l'insegnante che riportava indicazioni metodologiche sulle modalità di gestione della classe (lavoro individuale, in piccoli gruppi, discussioni collettive), sulle tempistiche e sui materiali.

La prima scheda di lavoro propone di far completare individualmente agli studenti dei grafi in cui ogni freccia rappresenta una relazione tra due numeri. Dopo il completamento si richiede agli studenti di mettere a confronto il proprio lavoro con quello di un compagno di classe per poi discutere con tutta la classe le osservazioni fatte.

Nella seconda scheda di lavoro si propone per la prima volta la rappresentazione con i barattoli per mettere a confronto una quantità (rappresentata da un solo barattolo) col proprio doppio (due barattoli). Allo studente viene chiesto sia di ipotizzare la numerosità di palline in un barattolo per poi determinare il numero di palline in due barattoli, sia di descrivere a parole la relazione tra due quantità A e B (una doppia dell'altra) utilizzando come soggetto una volta A e una volta B. Si chiede poi di confrontare il proprio lavoro con quello di un compagno e si propone al docente di introdurre il termine "relazione inversa". La terza scheda di lavoro richiede di confrontare una quantità rappresentata da un solo barattolo con un'altra rappresentata da un barattolo e una pallina. Si propone di lavorare in coppia a una traduzione simbolica di questa relazione e si propone all'insegnante di chiedere la descrizione di altri esempi usando le locuzioni "supera di ..." oppure "aumentata di ..." oppure "... in meno".

Nella quarta scheda viene riportata a parole la relazione "Le caramelle di Anna superano di 5 il doppio di quelle di Marco" e si chiede agli studenti di lavorare a coppie nella conversione di questa frase nella rappresentazione con barattoli e palline. Si chiede quindi di determinare alcune coppie di numeri che soddisfano la relazione. Si propongono alcuni esercizi di conversione da una rappresentazione all'altra e di sostituzione di particolari valori numerici.

La quinta scheda mostra una tabella a due colonne e si chiede agli studenti di individuare la relazione che lega i numeri che si trovano nella stessa riga (la stessa per tutte le righe della tabella). Tale relazione deve essere da loro espressa (lavorando in coppie) con diverse rappresentazioni, compresa la rappresentazione con barattoli e palline.

L'ultima scheda propone di individuare una coppia di numeri che soddisfa due relazioni espresse in forma simbolica (ovvero risolvere un semplice sistema di due equazioni di primo grado a due incognite). Viene proposto di convertire le relazioni nella rappresentazione con barattoli e palline per poi determinare il valore di ciascun barattolo.

Alle insegnanti sono stati forniti anche alcuni esercizi in modo che potessero utilizzarli come compito per casa per gli studenti. Essi sono stati costruiti in riferimento ai cinque aspetti dell'apprendimento della matematica (Fandiño Pinilla, 2008); in particolare, per quanto riguarda l'apprendimento e la gestione delle trasformazioni semiotiche, sono stati proposti esercizi di trattamento e conversione tra le diverse rappresentazioni - non solo riguardanti la rappresentazione con barattoli e palline.

\subsection{La prova}

Prima di iniziare il percorso e subito dopo il suo termine, tutte le classi (sia quelle del gruppo sperimentale, sia quelle del gruppo di controllo) hanno svolto la stessa prova composta da nove consegne. La prova è stata ideata dagli autori di questo contributo in modo che, nell'insieme, fossero valutate 
tutte le componenti dell'apprendimento della matematica descritte da Fandiño Pinilla (2008), così come viene indicato nelle descrizioni dei singoli quesiti presenti nella Tabella 1. Per ogni quesito si indicano le componenti dell'apprendimento principalmente indagate, pur essendo consapevoli che tutte le componenti concorrono nell'affrontare ciascuna consegna.

\begin{tabular}{|c|c|c|}
\hline Quesito & Testo della consegna & Componente apprendimento \\
\hline 1 & Completa le seguenti frasi con i numeri corretti. & Apprendimento algoritmico \\
\hline 2 & $\begin{array}{l}\text { Scrivi le relazioni rappresentate nelle figure come } \\
\text { nell'esempio } \\
\text { - } B \text { rispetto a } C \text { : "B è la metà di } C \text { " }\end{array}$ & $\begin{array}{l}\text { Apprendimento comunicativo } \\
\text { Apprendimento concettuale }\end{array}$ \\
\hline 3 & $\begin{array}{l}2 \cdot A+5=B \\
\text { Scrivi la relazione inversa utilizzando i simboli matematici. } \\
A=\end{array}$ & $\begin{array}{l}\text { Apprendimento algoritmico } \\
\text { Apprendimento concettuale }\end{array}$ \\
\hline 4 & $\begin{array}{l}\text { Completa il grafico inserendo le relazioni indicate dalle frecce. } \\
\text { Rappresenta il grafico con le frecce nella direzione inversa e } \\
\text { completalo indicando le relazioni. }\end{array}$ & $\begin{array}{l}\text { Apprendimento algoritmico } \\
\text { Apprendimento concettuale }\end{array}$ \\
\hline 5 & La somma di due numeri consecutivi è 85 . Di quali numeri si tratta? & Apprendimento strategico \\
\hline 6 & $\begin{array}{l}\text { Su una spiaggia ci sono } 120 \text { ombrelloni; gli ombrelloni aperti sono il } \\
\text { doppio di quelli chiusi. Quanti sono gli ombrelloni aperti e quanti } \\
\text { quelli chiusi sulla spiaggia? }\end{array}$ & Apprendimento strategico \\
\hline 7 & Collega con una freccia le rappresentazioni equivalenti. & $\begin{array}{l}\text { Apprendimento e gestione delle } \\
\text { trasformazioni semiotiche }\end{array}$ \\
\hline 8 & $\begin{array}{l}\text { Spiega utilizzando il linguaggio a parole cosa significa che la } \\
\text { differenza tra } A \text { e } B \text { è } 5 \text {. }\end{array}$ & Apprendimento comunicativo \\
\hline 9 & $\begin{array}{l}\text { Indica se le affermazioni scritte sono Vere }(\mathrm{V}) \text { o False (F). Motiva la scelta } \\
\text { proponendo un esempio numerico e correggi le affermazioni errate. }\end{array}$ & Apprendimento comunicativo \\
\hline
\end{tabular}

Tabella 1. Sintesi delle consegne presenti nella prova.

Il primo quesito richiede di completare frasi del tipo "21 è il triplo di ..." oppure "... diminuito di 11 è 22" inserendo il numero opportuno. Si possono completare le frasi anche solo ricorrendo al calcolo nel contesto numerico; pertanto questo quesito permette di valutare quantomeno l'apprendimento di tipo algoritmico.

Nel secondo quesito lo studente deve descrivere verbalmente relazioni tra quantità rappresentate con barattoli e palline (Figura 5). In particolare, si chiede di indicare cosa è $A$ rispetto $B$, cosa è $A$ rispetto $C$, cosa è $B$ rispetto ad $A$ e così via. Questo quesito permette di mettere in luce sia aspetti concettuali dell'apprendimento, sia aspetti comunicativi. In riferimento a questa consegna possiamo notare che la rappresentazione proposta non era stata presentata a nessuno degli studenti nella fase di pre-test ed era nota solo agli studenti del gruppo sperimentale nel post-test. A priori, possiamo quindi immaginare di notare notevoli differenze tra i due gruppi al termine dell'intervento sperimentale.

A

B

C

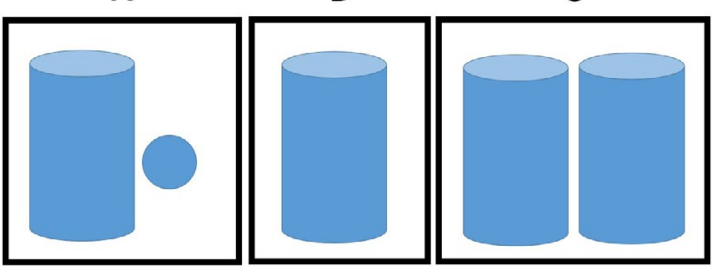

Figura 5. Quantità rappresentate con barattoli e palline nel secondo quesito della prova. 
Il terzo quesito propone di rappresentare la relazione inversa di $2 \cdot A+5=B$, mentre nel quarto quesito lo studente deve indicare quale relazione è rappresentata da ciascuna delle frecce presenti in un grafo (Figura 6). Viene poi chiesto di copiare lo stesso grafo invertendo il verso delle frecce e di indicare ancora una volta la relazione rappresentata da ciascuna freccia. Si tratta in entrambi i casi di quesiti che possono permettere di valutare la componente concettuale (in questo caso in riferimento al concetto di relazione inversa) e che potenzialmente possono mettere in luce anche aspetti algoritmici, soprattutto nel caso in cui si utilizzano soprattutto i numeri. In entrambi i casi si tratta di consegne familiari per gli studenti del gruppo sperimentale al termine dell'intervento; consegne analoghe non sono invece presenti nel libro di testo adottato e quindi dovrebbero presentare maggiori difficoltà per il gruppo di controllo.

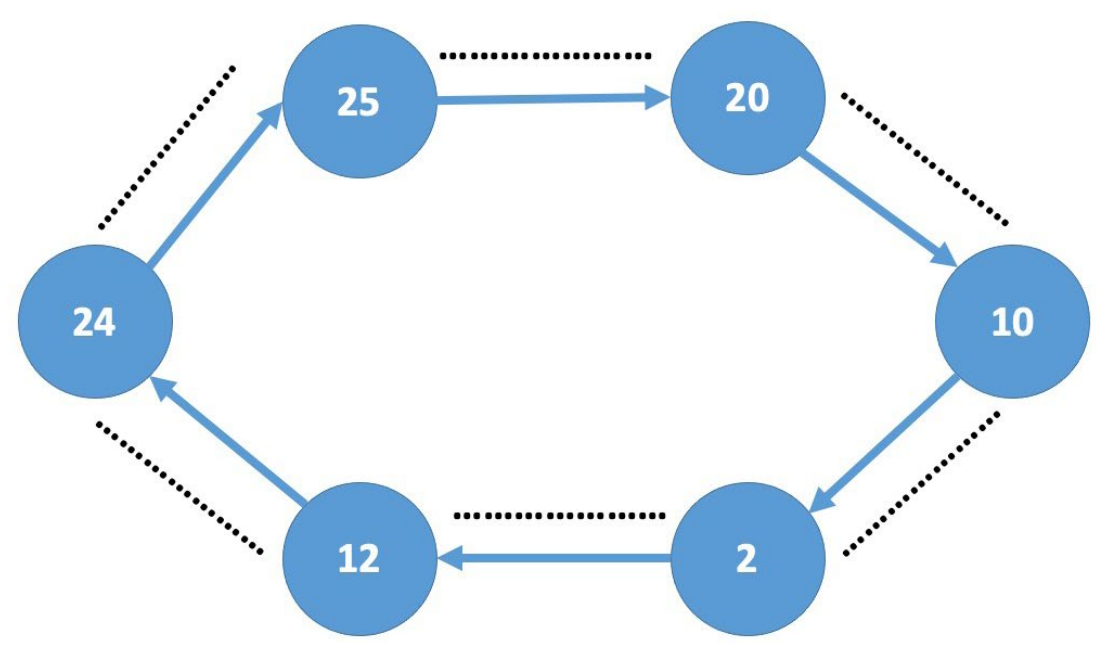

Figura 6. Grafo da completare nel quarto quesito della prova.

Il quinto e il sesto quesito si riferiscono a due situazioni problematiche, la prima in contesto matematico e la seconda in contesto extra-matematico. Tali quesiti sono pensati per valutare soprattutto la componente strategica dell'apprendimento. I testi delle due situazioni problematiche sono riportati, insieme a quelli delle altre consegne, nella Tabella 1. Situazioni simili sono presenti anche nel libro di testo adottato dalla scuola e quindi queste consegne dovrebbero essere familiari anche per il gruppo di controllo.

Il settimo quesito richiede di collegare con una freccia le rappresentazioni verbali matematicamente equivalenti di varie relazioni, per esempio "La somma tra $B$ e 5 è $A$ " e " $B$ aumentato di 5 è $A$ ". Allo studente è chiesto di individuare quali rappresentazioni si riferiscono alla stessa relazione; si indaga principalmente l'apprendimento e la gestione delle rappresentazioni semiotiche.

L'ottavo quesito richiede di spiegare verbalmente il significato dell'espressione "La differenza tra $A$ e $B$ è 5 ". Il focus è quindi sull'argomentazione, ovvero sull'apprendimento di tipo comunicativo. L'ultimo quesito viene risolto indicando se affermazioni del tipo "Tre volte $B$ è $A$, quindi un terzo di $A$ è $B^{\prime \prime}$ siano vere o false. Si tratta di un quesito che, in modo più semplice, indaga l'interpretazione del linguaggio verbale e richiede di prendere posizione su alcune affermazioni; si chiede anche di motivare la scelta. L'aspetto comunicativo è quindi prevalente.

La codifica delle risposte date dagli studenti è stata realizzata dal team di docenti della scuola. Ad ogni quesito è stato inizialmente attribuito un punteggio grezzo corrispondente al numero di completamenti o associazioni corrette. Successivamente tutti i punteggi sono stati normalizzati in modo da associare ad ogni quesito un valore compreso tra 0 e 1. 


\subsection{Analisi}

La prova di valutazione progettata ha lo scopo di valutare diversi aspetti dell'apprendimento di uno stesso contenuto matematico, pertanto dovrebbe presentare un'alta coerenza interna intesa come coerenza tra i singoli quesiti e il costrutto latente dell'intera prova. La coerenza interna è misurata attraverso il calcolo dell'alpha di Cronbach. Tale indice può essere considerato soddisfacente per ricerche esplorative quando il suo valore supera 0,70 (Nunnally \& Bernstein, 1994).

Per l'analisi dei dati raccolti nella prova di valutazione prima e dopo I'intervento didattico dobbiamo utilizzare un test non parametrico per via del numero ridotto di individui del campione. In particolare, abbiamo scelto il test di Mann-Whitney poiché si adatta a dati di tipo discreto che non prevedono a priori una distribuzione normale. L'ipotesi nulla che abbiamo assunto è che le distribuzioni delle performance dei due campioni messi a confronto siano uguali (sia nella prova complessiva sia nei singoli quesiti). Quando la probabilità che i due campioni siano uguali è inferiore al $5 \%$ (p-value $<0,05$ ), l'ipotesi può essere negata e cioè si può assumere che le performance dei due campioni siano significativamente diverse, altrimenti si interpretano i dati come se le differenze fossero dovute al caso. Applichiamo il test di Mann-Whitney ai risultati normalizzati della prova di valutazione somministrata prima dell'intervento didattico.

\section{Risultati}

Per quanto riguarda l'attendibilità della prova di valutazione, la coerenza interna è stata misurata attraverso il calcolo dell'alpha di Cronbach che è pari a 0,72. Questo risultato può essere considerato soddisfacente (Nunnally \& Bernstein, 1994) a fronte del basso numero di quesiti presenti nella prova. La media dei punteggi ottenuti dal gruppo sperimentale $(3,79)$ è più bassa di quella del gruppo di controllo $(5,91)$, ma il test di Mann-Whitney ci suggerisce che possiamo considerare i due campioni simili nonostante la differenza della media delle performance. Infatti, il test sulle performance dei singoli studenti non mostra differenze significative: la probabilità è maggiore del $5 \%$ ( $p$-value $=0,0655)$, quindi possiamo ipotizzare che le differenze riscontrate siano dovute al caso.

Successivamente abbiamo applicato il test Mann-Whitney confrontando le performance della prova di valutazione prima e dopo gli interventi didattici nelle due classi. Nelle Tabelle 2 e 3 sono mostrati i risultati dei due gruppi considerando sia la media dei punteggi dei singoli quesiti, sia della prova complessiva.

\begin{tabular}{|c|l|c|c|c|}
\hline Quesito & $\begin{array}{l}\text { Componente } \\
\text { apprendimento }\end{array}$ & $\begin{array}{l}\text { Punteggio medio } \\
\text { gruppo sperimenta- } \\
\text { le prima }\end{array}$ & $\begin{array}{l}\text { Punteggio medio } \\
\text { gruppo sperimenta- } \\
\text { le dopo }\end{array}$ & p-value \\
\hline 1 & Algoritmico & 0,82 & 0,91 & 0,2327 \\
\hline 2 & $\begin{array}{l}\text { Comunicativo } \\
\text { Concettuale }\end{array}$ & 0,19 & 0,76 & 0,00121 \\
\hline 3 & $\begin{array}{l}\text { Algoritmico - } \\
\text { Concettuale }\end{array}$ & 0,11 & 0,89 & 0,2611 \\
\hline 4 & $\begin{array}{l}\text { Algoritmico - } \\
\text { Concettuale }\end{array}$ & 0,70 & 0,48 & 0,0287 \\
\hline 5 & Strategico & 0,25 & 0,72 & 0,0096 \\
\hline 6 & Strategico & 0,24 & & 0 \\
\hline
\end{tabular}




\begin{tabular}{|c|l|c|c|c|}
\hline 7 & $\begin{array}{l}\text { Gestione delle } \\
\text { trasformazioni } \\
\text { semiotiche }\end{array}$ & 0,66 & 0,91 & 0,4364 \\
\hline 8 & Comunicativo & 0,10 & 0,41 & 0,0139 \\
\hline 9 & Comunicativo & 0,59 & 0,81 & 0,0301 \\
\hline $\begin{array}{l}\text { Media del punteggio } \\
\text { complessivo nella } \\
\text { prova }\end{array}$ & 3,79 & 6,44 & 0,0029 \\
\hline
\end{tabular}

Tabella 2. Tabella dei risultati nei singoli quesiti della prova di valutazione e p-value del test di Mann-Whitney del gruppo Sperimentale (in grigio sono evidenziati i quesiti in cui si riscontra un p-value $<5 \%$ ).

\begin{tabular}{|c|l|c|c|c|}
\hline Quesito & $\begin{array}{l}\text { Componente } \\
\text { apprendimento }\end{array}$ & $\begin{array}{l}\text { Punteggio medio } \\
\text { gruppo di controllo } \\
\text { prima }\end{array}$ & $\begin{array}{l}\text { Punteggio medio } \\
\text { gruppo di controllo } \\
\text { dopo }\end{array}$ & p-value \\
\hline 1 & Algoritmico & 0,84 & 0,87 & 0,4641 \\
\hline 2 & $\begin{array}{l}\text { Comunicativo } \\
\text { Concettuale }\end{array}$ & 0,27 & 0,38 & 0,3446 \\
\hline 3 & $\begin{array}{l}\text { Algoritmico - } \\
\text { Concettuale }\end{array}$ & 0,50 & 0,80 & 0,4207 \\
\hline 4 & $\begin{array}{l}\text { Algoritmico - } \\
\text { Concettuale }\end{array}$ & 0,77 & 0,77 & 0,1611 \\
\hline 5 & Strategico & 0,48 & 0,61 & 0,2611 \\
\hline 6 & Strategico & 0,39 & 0,81 & 0,1949 \\
\hline 8 & $\begin{array}{l}\text { Gestione delle } \\
\text { trasformazioni } \\
\text { semiotiche }\end{array}$ & 0,91 & 0,51 & 0,1401 \\
\hline $\begin{array}{l}\text { Comunicativo } \\
\text { Media del punteggio } \\
\text { prova }\end{array}$ & Comunicativo & 0,38 & 0,82 & 0,4522 \\
\hline
\end{tabular}

Tabella 3. Tabella dei risultati nei singoli quesiti della prova di valutazione e p-value del test di Mann-Whitney del gruppo di Controllo.

Consideriamo prima la media dei punteggi complessivi della prova: il gruppo sperimentale mostra una media dei punteggi significativamente aumentata (da 3,79 a 6,44); al contrario, il gruppo di controllo non mostra differenze significative (da 5,91 a 5,90), entrambi i risultati sono confermati dal test di Mann-Whitney (per il gruppo sperimentale p-value =0,0029; per il gruppo di controllo $p$-value $=0,1587$ ). Nel dettaglio dei quesiti, si può notare che nel gruppo sperimentale si osservano differenze significative in più della metà dei quesiti, anche se non nella totalità. II gruppo di controllo non sembra invece aver fatto progressi significativi per nessuno dei quesiti.

\section{Discussione e conclusioni}

Abbiamo presentato un'analisi di una particolare rappresentazione grafica intermedia tra situazioni problematiche e simbolismo algebrico. Tale rappresentazione è stata incorporata in una sequenza 
didattica sperimentata in classi prime medie. Per valutare l'efficacia dell'intervento è stata predisposta una prova ideata in modo da essere rappresentativa di tutte le componenti dell'apprendimento della matematica individuate da Fandiño Pinilla (2008); la prova ha dimostrato soddisfacenti capacità misuratorie in termini di coerenza interna. I risultati mostrano che il gruppo sperimentale presenta differenze significative nel punteggio medio tra pre- e post-test, cosa che non accade per il gruppo di controllo.

Prendendo in esame le singole componenti della prova, possiamo osservare che in nessun quesito il gruppo di controllo sembra aver avuto differenze significative (Tabella 3) mentre il gruppo sperimentale le mostra in più della metà dei quesiti (Tabella 2). Un importante aspetto da notare prima di passare all'analisi dettagliata dei quesiti, è che essi (fatta eccezione per il quesito numero 2) non presentano rappresentazioni con barattoli e palline, ma sono privilegiate rappresentazioni che utilizzano il linguaggio verbale, simbolico, tabulare e grafico.

Entrando nel dettaglio, le maggiori differenze si riscontrano in tutti i quesiti legati alla componente strategica (5 e 6), alla maggior parte di quelli relativi alla componente comunicativa (8 e 9) e ad uno legato a quella concettuale (3). I quesiti 5 e 6 afferiscono alla componente strategica e presentano due situazioni: il primo in contesto matematico e il secondo in contesto reale. L'aspetto di notevole interesse è che, tra i due, il quesito in cui si registrano le maggiori differenze (con una significatività statistica maggiore) è quello in contesto reale e quindi quello che prevede, oltre all'attivazione di un processo di matematizzazione verticale, anche un processo di matematizzazione orizzontale (Treffers, 1987).

I quesiti 8 e 9, invece, si riferiscono alla componente comunicativa: in particolare, il primo chiede di descrivere con il linguaggio verbale una relazione tra due quantità, mentre il secondo chiede di verificare e motivare se delle affermazioni sono vere o false. Nell'ambito della componente comunicativa, non si sono riscontrate differenze significative nel quesito 2 poiché coinvolge aspetti comunicativi che in generale non si riferiscono ad argomentazioni (spiegazioni o giustificazioni nel senso di Brunero e Panero, 2019).

Infine, il quesito 3 chiede di manipolare un'espressione scritta con il linguaggio algebrico per determinare la relazione inversa. Il quesito è stato categorizzato all'interno delle componenti di apprendimento concettuale e algoritmico; coinvolgendo il concetto di relazione inversa, potrebbe non essere stato familiare per il gruppo di controllo e quindi il vantaggio del gruppo sperimentale in questo caso potrebbe essere proprio nell'apprendimento concettuale.

In conclusione, le differenze maggiori si riscontrano negli apprendimenti strategici e comunicativi che potrebbero confermare lo stretto rapporto che esiste tra risoluzione di problemi e argomentazione. Come osserva Di Martino

\begin{abstract}
«Problem solving e argomentazione [...] sono ovviamente tra loro collegati: per valutare la risoluzione di un problema dobbiamo avere informazioni sia sui processi attivati (quindi è necessaria la spiegazione) sia valutare le giustificazioni delle scelte fatte (quindi la vera e propria argomentazione). D'altra parte richiedere di argomentare ha senso laddove lo studente è chiamato a fare delle scelte, ad assumersi delle responsabilità nell'attivazione dei processi di pensiero, e dunque in merito a processi produttivi».
\end{abstract}

(Di Martino, 2017, p. 25)

Un approccio di questo tipo potrebbe fare la differenza nello sviluppare e stimolare aspetti trasversali legati all'argomentazione e alla risoluzione di problemi. Lo stesso Piano di studio della scuola dell'obbligo ticinese (DECS, 2015) suggerisce il ricorso a situazioni di apprendimento significative che consentano di lavorare sulla capacità di utilizzare concetti, principi e metodi della matematica per comprendere, spiegare, esaminare e rappresentare la realtà. Ricordiamo, inoltre, che gli aspetti di competenza "comunicare e argomentare" e "matematizzare e modellizzare" sono due dei quattro processi individuati per la matematica nel Piano di studio della scuola dell'obbligo ticinese (DECS, 2015). 
Questi risultati fanno eco alla vasta letteratura che mette in evidenza come l'approccio standard che si concentra solo sull'apprendimento concettuale e algoritmico si può superare (Fandiño Pinilla, 2008). L'attivazione di processi riproduttivi (per esempio la risoluzione di esercizi) può essere accompagnata da processi produttivi (risoluzione di problemi e argomentazione), dando una rilevanza ai processi e alla capacità di saperli descrivere e sostenere (argomentazione) piuttosto che ai prodotti (risultati). Questo vale tanto nel processo di insegnamento-apprendimento in generale quanto nella fase valutativa in particolare.

Dobbiamo anche notare i limiti dello studio qui presentato: il numero di studenti coinvolti è sicuramente basso e il fatto che appartengano tutti alla stessa scuola non ci permette di avere nessuna informazione su come una variazione di contesto potrebbe influire sui risultati della sperimentazione. Dal punto di vista quantitativo lo studio potrebbe essere rafforzato attraverso un diverso campionamento dei partecipanti. Ulteriori sviluppi potrebbero essere effettuati anche da un punto di vista qualitativo. Per esempio, abbiamo notato differenze nei quesiti di carattere argomentativo che potrebbero dipendere proprio dal fatto di aver richiesto una spiegazione in un caso e una giustificazione nell'altro. Un'analisi delle specifiche argomentazioni prodotte dagli studenti potrebbe permettere di identificare le differenze per queste tipologie di argomentazione. Inoltre, un'analisi qualitativa dei protocolli relativi alle situazioni problematiche potrebbe permettere di meglio descrivere il ruolo delle diverse rappresentazioni nel processo di soluzione dei problemi.

In conclusione, ai risultati quantitativi che abbiamo presentato nelle sezioni precedenti, aggiungiamo alcune considerazioni che abbiamo condiviso con i docenti coinvolti in questa esperienza: una prima osservazione riguarda il fatto che tutti i ragazzi della classe sono riusciti a partecipare alle attività proposte senza particolari difficoltà; questo testimonia il potenziale della proposta in termini di inclusività. Più di un insegnante ha notato che già nel post-test una buona parte degli studenti ha abbandonato spontaneamente la rappresentazione con barattoli e palline; questo suggerisce che essa si presta molto bene come rappresentazione intermedia che, per definizione (Davydov, 1982), deve essere superata. Infine, I'approccio proposto ha suscitato un particolare entusiasmo non solo tra gli studenti ma anche tra i loro insegnanti. Da un punto di vista di metodologia della ricerca, tale entusiasmo potrebbe essere ascrivibile come una delle cause dei risultati ottenuti; se invece ci mettiamo nel punto di vista dell'implementazione in classe, esso fornisce una buona ragione per adottare questo cambiamento di paradigma. L'unico limite riportato dagli insegnanti coinvolti riguarda le tempistiche di implementazione che richiedono una ristrutturazione della programmazione.

\section{Ringraziamenti}

Si ringraziano Antonella Castellini e Chiara Giberti per la proficua collaborazione nella progettazione della proposta didattica e della prova di valutazione. Si ringraziano inoltre tutte le docenti della scuola secondaria di primo grado dell'I.C. "Guido Novello" di Ravenna per l'entusiasta collaborazione e le profonde riflessioni condivise: Loredana Adamo, Laura Caruso, Loretta Evangelisti, Francesca Focaccia, Alessandra Gennari.

\section{Bibliografia}

Bazzini, L., \& laderosa, R. (2000). Approccio all'Algebra. Riflessioni didattiche. FrancoAngeli.

Boyer, C. B. (2017). Storia della matematica. Mondadori. (Titolo originale: A History of Mathematics pubblicato nel 1968).

Brunero, A. M., \& Panero, M. (2019). Sviluppare e valutare competenze argomentative in matematica: un percorso per la scuola elementare. Didattica della matematica. Dalla ricerca alle pratiche d'aula, 6, 82-108. 
Cai, J., Lew, H. C., Morris, A., Moyer, J. C., Fong Ng, S., \& Schmittau, J. (2005). The development of students' algebraic thinking in earlier grades: Lessons from China and Singapore. ZDM, 37(1), 5-15.

Castellini, A. (2016). Against problem solving by segment method. EDiMaST: Experiences of Teaching with Mathematics, Sciences and Technology, 2(2), 287-302.

D’Amore, B., \& Sbaragli, S. (2017). La matematica e la sua storia. Dalle origini al miracolo greco. Edizioni Dedalo.

Davydov, V. V. (1982). The psychological characteristics of the formation of elementary mathematical operations in children. In T. P. Carpenter, J. M. Moser \& T. A. Romberg (Eds.), Addition and Subtraction: A Cognitive Perspective (pp. 224-238). Routledge.

Di Martino, P. (2017). Problem solving e argomentazione matematica. Didattica della matematica. Dalla ricerca alle pratiche d'aula, 1, 23-37.

Dipartimento dell'educazione, della cultura e dello sport. (2015). Piano di studio della scuola dell'obbligo ticinese. http://www.pianodistudio.ch/

Duval, R. (2008). Eight problems for a semiotic approach in mathematics education. In L. Radford, G. Schubring \& F. Seeger (Eds.), Semiotics in mathematics education (pp. 39-61). Brill Sense.

Fandiño Pinilla, M. I. (2008). Molteplici aspetti dell'apprendimento della matematica. Erickson.

Fandiño Pinilla, M. I. (2015). Insegnare e valutare la competenza in Matematica. In AA. VV. (2015). Didattica per competenze. Supplemento a La Vita Scolastica, 70(2), 10-14.

Fischbein, E., Deri, M., Nello, M. S., \& Marino, M. S. (1985). The role of implicit models in solving verbal problems in multiplication and division. Journal for research in mathematics education, 16(1), 3-17.

Fischbein, H. (1987). Intuition in science and mathematics: An educational approach. Springer Science \& Business Media.

Kieran, C. (1981). Concepts associated with the equality symbol. Educational Studies in Mathematics, 12(3), 317-326.

Mellone, M., Baccaglini-Frank, A., \& Martignone, F. (2020). Measuring Rice in Early-Childhood Education Activities: A Bridge Across Discrete and Continuous Magnitudes. In M. Carlsen, I. Erfjord \& P. S. Hundeland (Eds.), Mathematics Education in the Early Years (pp. 161-176). Springer.

Ministero dell'Istruzione, dell'Università e della Ricerca. (2012). Indicazioni nazionali per il curricolo della scuola dell'infanzia e del primo ciclo di istruzione. http://www.indicazioninazionali.it/

Navarra, G. (2019). Il progetto ArAl per un approccio relazionale all'insegnamento nell'area aritmetico-algebrica. Didattica della matematica. Dalla ricerca alle pratiche d'aula, 5, 70-94.

Nunnally, J. C., \& Bernstein, I. H. (1994). Psychometric Theory (3rd ed.). McGraw-Hill.

Polotskaia, E., \& Savard, A. (2021). Some multiplicative structures in elementary education: a view from relational paradigm. Educational Studies in Mathematics, 106(3), 447-469.

Radford, L. (2008). Towards a cultural theory of learning. In L. Radford, G. Schubring \& F. Seeger (Eds.), Semiotics in mathematics education: Epistemology, history, classroom, and culture (pp. 215-234). Brill Sense.

Radford, L. (2010). The eye as a theoretician: Seeing structures in generalizing activities. For the learning of mathematics, 30(2), 2-7.

Sbaragli, S. (2005). Misconcezioni "inevitabili" e misconcezioni "evitabili". La matematica e la sua didattica, 1, 57-71. 
Siety, A. (2003). Matematica, mio terrore. Alla scoperta del lato umano della matematica. Salani Editore. (Titolo originale: Mathématiques, ma chère terreur pubblicato nel 2001).

Steffe, L. P. (1994). Children's multiplying schemes. In G. Harel \& J. Confrey (Eds.), The development of multiplicative reasoning in the learning of mathematics (pp. 1-41). SUNY.

Treffers, A. (1987). Three dimensions. A model of goal and theory description in mathematics instruction-The Wiskobas project. Kluwer Academic Publishers.

Xin, Y. P., Zhang, D., Park, J. Y., Tom, K., Whipple, A., \& Si, L. (2011). A comparison of two mathematics problem-solving strategies: Facilitate algebra-readiness. The Journal of Educational Research, 104(6), 381-395.

Yackel, E. (2001). Explanation, justification and argumentation in mathematics classrooms. In M. Van den Heuvel-Panhuizen (Ed.), Proceedings of the 25th Conference of the International Group for the Psychology of Mathematics Education PME-25 vol. 1 (pp. 1-9). Utrecht University. 